\title{
Comparison of Srs-24 And Srs-22 Scores in Thirty Eight Adolescent Idiopathic Scoliosis Patients Who Had Undergone Surgical Correction
}

\author{
CYW Chan, MS Ortho, LB Saw, MS Ortho, MK Kwan, MS Ortho \\ Department of Orthopaedic Surgery, University Malaya Medical Centre, Kuala Lumpur, Malaysia
}

\begin{abstract}
Adolescent idiopathic scoliosis is a spinal deformity that affects patients' self image and confidence. Surgery is offered when the curvature is greater than 50 degrees based on the likelihood of curvature progression. Outcome measures for scoliosis correction can be described in terms of radiological improvement or improvement of health related quality of life scores. The Scoliosis Research Society 22 (SRS-22) and Scoliosis Research Society 24 (SRS-24) questionnaires are widely accepted and used to characterize clinical results. Therefore, this prospective study of 38 patients aims to investigate how the SRS-24 and SRS-22 questionnaires compare to each other in terms of scoring when the same group of patients is evaluated. The SRS-22 questionnaire tends to give an inflated value in the overall score, pain and self image domain compared to the SRS-24 questionnaire.
\end{abstract}

Key Words:

SRS-22, SRS-24, Adolescent Idiopathic Scoliosis

\section{INTRODUCTION}

Surgical treatment of scoliosis is indicated for patients with curvature more than fifty degrees ${ }^{1}$. The aim is to halt curve progression but frequently for patients, improved cosmetic outcome with surgical correction is what drives them to undertake this operation. Outcome measures for defining success of the operation can be radiological or clinical. While objective measurement of curve correction through radiological methods has been widely reported, this does not necessarily lead to enhanced patient satisfaction. Questionnaires to evaluate functional and psychosocial outcome for spine related conditions are aplenty but Haher et al pioneered a disease specific, health-related quality of life questionnaire for scoliosis patients which was popularly known later as the Scoliosis Research Society 24 (SRS-24) questionnaire $^{2}$. Although it is simple and widely accepted, there were some weaknesses of the questionnaire. Asher et al. note that there is a lack of internal consistency in the function domain, unknown test-retest reliability and unknown criteria and discriminant validity ${ }^{3}$. With this, a modification of the SRS-24 questionnaire was introduced in the form of the Scoliosis Research Society 22 (SRS-22) questionnaire. Therefore, this study aims to investigate how the SRS-24 and SRS-22 questionnaire compare to each other in terms of scoring when the same group of patients was evaluated.

\section{MATERIALS AND METHODS}

This is a prospective study involving thirty-eight adolescent idiopathic scoliosis patients who underwent surgery at our institution from January 2001 to December 2005, all of whom had post-operative follow up of more than one year. The English version of the SRS-24 and SRS-22 questionnaires was used. Of the twenty two questions in the SRS-22 questionnaire; when compared to the SRS-24 questionnaire, ten are exactly the same, six has been modified while there were six completely new questions which were actually adaptation from the SF-36 questionnaires.

Besides the Mental Health domain, other domains in the SRS 22 questionnaire were present in the SRS 24 questionnaire; Pain, Self Image, Function, and Satisfaction. The additional domains present in the SRS-24 questionnaire are Activity, Post-operative function and Post-operative Image domain. However, the questions which represent these domains might not be the same as in the SRS-22. For example, when comparing the Pain Domain of SRS-24 and SRS-22, question 1,2,6 and 8 in SRS 24 were present in the same domain in SRS-22, but question 3 and 11 in the SRS 24 questionnaire have been moved to the self image domain in the SRS-22 questionnaire. Question 18 in the pain domain of SRS-24 has been omitted from SRS-22.

These two questionnaires were tested on the group of thirty eight patients by two independent evaluators at two different sittings. All patients had acceptable command of the English language but minor translations and explanation was allowed. The curvature patterns for this group of patients were classified using the King and Moe's classification ${ }^{4}$. 
Table I: Values for thoracic, lumbar and major Cobb angle, before and after surgery

\begin{tabular}{|lllr|}
\hline Cobb angle & $\mathbf{n}$ & Mean \pm SD (o) & Range (o) \\
\hline Pre-operative thoracic curvature & 31 & $68.1 \pm 23.40$ & $32-123$ \\
Post-operative thoracic curvature & 31 & $39.5 \pm 18.17$ & $14-100$ \\
Pre-operative lumbar curvature & 23 & $59.4 \pm 17.99$ & $32-106$ \\
Post-operative lumbar curvature & 23 & $27.0 \pm 14.43$ & $7-63$ \\
Pre-operative major curvature & 38 & $68.6 \pm 20.17$ & $42-123$ \\
Post-operative major curvature & 38 & $35.8 \pm 19.19$ & $7-100$ \\
\hline
\end{tabular}

$\mathrm{n}$ : number of subjects

Table II: Distribution of scores according to domains of the SRS-22 and SRS-24 questionnaires.

\begin{tabular}{|lcclcc|}
\hline & $\begin{array}{c}\text { SRS-22 } \\
\text { Domains }\end{array}$ & & SRS-24 \\
Mean \pm SD (o) & Range (o) & Domains & Mean \pm SD (o) & Range (o) \\
\hline Self Image * & $4.4 \pm 0.51$ & $3.2-5.0$ & Pain * & $4.2 \pm 0.54$ & $3.3-5.0$ \\
Function/Activity & $3.9 \pm 0.69$ & $2.8-5.0$ & Self image * & $3.4 \pm 0.56$ & $2.0-4.7$ \\
& $3.8 \pm 0.52$ & $2.8-5.0$ & Function & $3.7 \pm 0.72$ & $2.0-4.7$ \\
Satisfaction with & & & Activity & $3.8 \pm 0.90$ & $2.0-5.0$ \\
management & $4.3 \pm 0.79$ & $1.4-5.0$ & Satisfaction with management & $4.2 \pm 0.71$ & $2.3-5.0$ \\
Mental health & $4.3 \pm 0.75$ & $3.0-5.0$ & Post-op Image & $3.8 \pm 0.78$ & $2.0-5.0$ \\
& & & Post-op function & $3.0 \pm 1.30$ & $1.0-5.0$ \\
Overall score * & $4.2 \pm 0.37$ & $3.4-4.9$ & Overall score * & $3.8 \pm 0.38$ & $3.0-4.9$ \\
\hline
\end{tabular}

* Domains where differences are statistically significant with $p<0.05$

Curve magnitude was quantified using Cobb's method. Data was entered into the SPSS programme, and differences in the scores were tested for significance using the paired sample ttest. The level of significance was set at $\mathrm{p}<0.05$.

\section{RESULTS}

There were thirty-two females and six males in our study. Twenty-six of these patients $(68.4 \%)$ were Chinese, eight $(21.1 \%)$ were Malays and four were Indian patients (10.5\%). The age of the patients ranged from twelve to twenty eight years old with a mean age of $18.4 \pm 3.5$ years old. The mean follow up duration was thirty-nine months (range, 12 to 90 months). The most common curve type was King's 3 curves; this curve type was present in sixteen patients (42.1\%). There were seven patients (18.4\%) with King's type 1 curve, seven (18.4\%) with King's type 2 and one with King's type 4 $(2.6 \%)$. The curves of seven patients $(18.4 \%)$ were not classifiable under the King's classification as they were thoracolumbar or lumbar curves which would fall into the Lenke 5C category. The curve magnitudes for the patients are shown in Table I. The mean pre-operative Cobb angle is 68.60 and the mean post-operative Cobb angle is 35.80 , representing a mean correction rate of $48.5 \%$. Twenty three patients were corrected using a polyaxial pedicle screw system and thirteen patients using hybrid instrumentation. Two patients underwent combined anterior and posterior surgery.

Table II shows the scores of the thirty-eight patients when interviewed using both SRS-22 and SRS-24 questionnaire. The overall score of the two questionnaires show statistically significant differences with SRS-22 having a score of 4.2 compared to a score of 3.8 when SRS-24 is used. The Self Image domain and Pain domain in these two questionnaires are also significantly different.

Despite these differences, the two questionnaires do show some similar trends. The domains that score the highest on both questionnaires are the Pain and Satisfaction with Management domain. The Function/ Activity in the SRS-22 questionnaire and the Function and Activity domain in the SRS-24 questionnaire have very similar scores.

The two domains that are absent from the SRS-22 questionnaire are Post-op Image and Post-op Function. When these scores are compared with the general function and self image scores, there is a significant decrease in the post-op function scores while the post-op self image scores increased significantly. The Mental Health domain in the SRS-22 has a mean score of 4.3 .

\section{DISCUSSION}

Surgical correction of adolescent idiopathic scoliosis has progressed much with improvement in instrumentation methods. Use of pedicle screw systems has enabled surgeons to increase the amount of correction ${ }^{5,67}$, however, measuring a patient's psychosocial status and satisfaction with outcome of surgery is probably more pertinent. These are aspects that are evaluated in the SRS- 24 questionnaire. This questionnaire was first introduced by Haher et al in 1999 whereby he tested these twenty-four shortlisted questions in 244 patients at multiple centers. The questions were shown to 
have a high degree of reliability except for the Function domain. The questions were also able to discriminate between normal subjects and subjects with scoliosis ${ }^{2}$. This questionnaire was then widely used by surgeons to report their clinical results.

In 2006, Crawford et al reported the clinical results of patients who underwent anterior endoscopic scoliosis surgery using the SRS-24 questionnaire ${ }^{8}$. Other surgeons who have reported their results using this questionnaire are Newton et al, Merola et al, Sweet et al and Watanabe et al $9,10,11,12$. The pattern of scores in our study was similar to Crawford et al, Merola et al and Newton et al whereby postop function of patients generally deteriorated but other domains showed satisfactory scores ${ }^{8,9,10}$. In 2007, Watanabe et al published results using the SRS 24 questionnaire and found correlation between the magnitude of curve correction and curve rotation and patient's self image.

However, the SRS-24 questionnaire is not without weaknesses. Sanford et al, in 1999, found that there was lack of internal consistency in the function-activity domain. He attributed this to question 10 and 11, which he thought correlated better with the pain domain. He also found that among the three questions in the Satisfaction with Management domain, question 23 had low consistency ${ }^{13}$.

Following this study, in 2000, Asher et al further refined the SRS-24 questionnaire. He removed questions 16 to 21 as they requires patient recall, and question 23 because of its low reliability. Questions 9,11,12,13 in SRS-24 were expanded so that there were two to five possible responses. The function and activity domains were combined. In addition, a separate domain was created, mental health domain, which is actually an adaptation from the SF-36 question 3. Following this, the SRS-22 questionnaire underwent extensive validation in several studies documenting its reliability, concurrent validity, discriminant validity and sensitivity to change ${ }^{14,15,16,17}$. The SRS-22 questionnaire was also shown to correlate well with the SF36 questionnaire ${ }^{18}$. Turkish and Spanish versions of this questionnaire have also been validated ${ }^{19,20}$.
Despite the fact that SRS-22 is a refinement of SRS-24 and has undergone more validation studies, both SRS-22 and SRS-24 questionnaire are still widely used to report clinical results. One of the aims of scoring patients with health related a questionnaire is to enable surgeons to compare clinical results. Therefore the aim of this study was to investigate whether there is any difference in the scores of the two questionnaires when administered to the same group of patients. This would also help us take into context the value of the scores when the questionnaire is used as a method to compare different treatment methods.

We found significant differences between the overall score, the pain domain and the self image domain of the SRS-22 and SRS-24 questionnaires. The SRS-24 values were significantly lower compared to the SRS-22 values for these categories. If the scores were used to compare clinical results, the SRS-22 questionnaires will lead to an inflated value these three domains. Another useful method of measuring clinical outcome is the use of the post-op image and function domain in the SRS-24 questionnaire. Improvement or deterioration in scores can be a useful indicator to the success of the operation.

There are some limitations in this study. The study population is a multicultural group and translation of the questions into their respective dialect is sometimes unavoidable. This might affect the reliability of the responses given by the patient. In addition, a larger study population would give more representative scores.

\section{CONCLUSION}

We conclude that when SRS-22 and SRS-24 questionnaires are used to compare clinical results of various treatment methods, the value of the scores of these two questionnaires cannot be used interchangeably despite the similarity in the questions and the domains. The SRS-22 questionnaire tends to give an inflated value in the overall score, pain and self image domain when compared to the SRS-24 questionnaire. 


\section{REFERENCES}

1. Weinstein SL. Idiopathic scoliosis: natural history. Spine 1986;11: 780-3.

2. Haher TR, Gorup JM, Shin TM, Homel P, Merola AA, Grogan DP, et al. Results of the scoliosis research society instrument for evaluation of surgical outcome in adolescent isiopathic scoliosis. Spine 1999; 24: 1435-40.

3. Asher MA, Lai SM, Burton DC. Further development and validation of the scoliosis research society (SRS) outcomes instrument. Spine 2000; 25: 2381-86.

4. King HA, Moe JH, David SB, Winter RB. The selection of fusion levels in thoracic idiopathic scoliosis. J Bone Joint Surg 1983; 65: 1302-13.

5. Dobbs MB, Lenke LG, Kim YJ, Kamath G, Peelle MW, Bridwell KH. Selective posterior thoracic fusions for adolescent idiopathic scoliosis: comparison of hooks versus pedicle screws. Spine 2006; 31: 2400-4.

6. Suk S, Lee CK, Kim W, Chung Y, Park Y. Segmental pedicle screw fixation in the treatment of thoracic idiopathic scoliosis. Spine 1995; 20: 1399-405.

7. Kim YJ, Lenke LG, Kim J, Bridwell KH, Cho SK, Cheh G, et al. Comparative analysis of pedicle screw versus hybrid instrumentation in posterior spinal fusion of adolescent idiopathic scoliosis. Spine 2006; 31: 291-8.

8. Crawford JR, Izatt MT, Adam CJ, Labrom RD, Askin GN. A prospective assessment of SRS-24 scores after endoscopic anterior instrumentation for scoliosis. Spine 2006; 31: E817-22.

9. Newton PO, Parent S, Marks M, Pawelek J. Prospective evaluation of 50 consecutive scoliosis patients surgically treated with thoracoscopic anterior instrumentation. Spine 2005; 30: S100-9.

10. Merola AA, Haher TR, Brkaric M, Panagopoulos G, Mathur S, Kohani O, et al. A multicenter study of the outcomes of the surgical treatment of adolescent idiopathic scoliosis using the scoliosis research society (SRS) outcome instrument. Spine 2002; 27: 2046-51.

11. Sweet FA, Lenke LG, Bridwell KH, Blanke KM, Whorton J. Prospective radiographic and clinical outcomes and complications of single solid rod instrumented anterior spinal fusion in adolescent idiopathic scoliosis. Spine 2001; 26: 1956-65.

12. Watanabe K, Hasegawa K, Hirano T, Uchiyama S, Endo N. Evaluation of postoperative residual spinal deformity and patient outcome in idiopathic scoliosis patients in japan using the scoliosis research society outcomes instrument. Spine 2007; 32: 550-54.

13. White SF, Asher MA, Lai SM, Burtom DC. Patients' perceptions of overall function, pain, and appearance after primary instrumentation and fusion for idiopathic scoliosis. Spine 1999; 24: 1693-700.

14. Asher M, Min Lai S, Burton D, Manna B. The reliability and concurrent validity of the Scoliosis Research Society-22 patient questionnaire for idiopathic scoliosis. Spine 2003; 28: 63-9.

15. Berven S, Deviren V, Demir-Deviren S, Hu SS, Bradford DS. Studies in the modified scoliosis research society outcomes instrument in adults: validation, reliability, and discrimination capacity. Spine 2003; 28: 2164-9.

16. Asher M, Lai SM, Burton DC, Manna BJ. Discrimination validity of the scoliosis research society-22 patient questionnaire: relationship to idiopathic scoliosis curve pattern and curve size. Spine 2003; 28: 74-7.

17. Asher M, Lai SM, Burton D, Manna B. Scoliosis research society-22 patient questionnaire: responsiveness to change associated with surgical treatment. Spine 2003; 28: 70-3.

18. Lai SM, Asher M, Burton D. Estimating srs-22 quality of life measures with SF-36. Application in idiopathic scoliosis. Spine 2006; 31: 473-8.

19. Bago J, Climent J, Ey A, Perez-Grueso FJ, Izquierdo E. The Spanish version of the srs-22 patient questionnaire for idiopathic scoliosis: transcultural adaptation and reliability analysis. Spine 2004; 29: 1676-80.

20. Alanay A, Cil A, Berk H, Acaroglu RE, Yazici M, Akcali O, et al. Reliability and validity of adapted Turkish version of scoliosis research society-22 (srs-22) questionnaire. Spine 2005; 30: 2464-8. 